\title{
Molecular Type Spread Molecular Shift Keying for Multiple-Access Diffusive Molecular Communications
}

\author{
Weidong Gao, Student Member, IEEE, Terrence Mak, Senior Member, IEEE, and Lie-Liang Yang, Fellow, IEEE
}

\begin{abstract}
In nano-networking, many nano-machines need to share common communication media, in order to achieve information exchange and data fusion. Multiple-access is an important technique for multiple nano-machines to send information to one access point (AP) or fusion center. Built on the Molecular Shift Keying (MoSK) modulation, this paper proposes a novel Molecular Type Spread MoSK (MTS-MoSK) scheme for achieving multiple-access transmission in diffusionbased molecular communications (DMC). Correspondingly, two detection schemes are introduced and investigated, which are the Maximum Selection assisted Majority Voter Detection (MSMVD) and Equal-Gain Combining Detection (EGCD). The error performance of the MTS-MoSK DMC systems with respectively the two detection schemes is analyzed, when both MultipleAccess Interference (MAI) and Inter-Symbol Interference (ISI) are taken into account. Furthermore, the performance of MTSMoSK DMC systems is investigated and compared with the aid of analytical results as well as Monte-Carlo and particlebased simulations. Our studies and performance results show that MTS-MoSK constitutes a promising candidate for implementing multiple-access DMC, and MS-MVD has the potential to outperform EGCD in DMC.
\end{abstract}

Index Terms-Diffusive molecular communications, molecular shift keying, molecular type spreading, multiple-access, majority vote, equal gain combining, inter-symbol interference, error performance analysis.

\section{INTRODUCTION}

In order to boost the capability of nano-networks, the individual nano-machines of each being only able to complete simple tasks are required to cooperate with each other and/or to exchange information, so that more complex mission can be fulfilled. For example, in a molecular sensor network, each molecular sensor has only limited storage space and computation potential, and can only observe a very limited region. In order to monitor a relatively large region, multiple and, possibly, many molecular sensors may have to be distributed over the region in order to attain a seamless coverage. Furthermore, considering the limited storage and computation capabilities of individual sensors, the observations obtained by individual sensors may need to be conveyed to a fusion center, where the final decision about the monitored event is made. In the above described process, one of the important challenges is how to send the observations obtained by individual sensors to the fusion center, efficiently and reliably. Explicitly, this task can be achieved with the aid of the multiple-access techniques, which deal with information transmission between

W. Gao, T. Mak and L.-L. Yang are with School of Electronics and Computer Science, University of Southampton, SO17 1BJ, UK. (E-mail: 1s25g12, tsm3g15, 1ly@ecs.soton.ac.uk, http://www-mobile.ecs.soton.ac.uk/lly). multiple transmit nodes (like molecular sensors) and one common receive node (like fusion center). Hence, significant research effort is required for designing the efficient multipleaccess schemes for supporting nano-networking. In this paper, we propose and investigate a multiple-access scheme for Diffusion-based Molecular Communications (DMC).

In literature, there are several multiple-access transmission techniques having been proposed in the context of DMC. Specifically, the Molecular Code-Division Multiple-Access (MCDMA) introduced in [1,2] allows multiple nano-machines to simultaneously transmit information to a receiver by assigning different nano-machines unique signature codes. These transmitter-specific signature codes are used by the receiver to distinguish the information received from different transmit nano-machines. In [3-5], Molecular Time-Division MultipleAccess (MoTDMA) has been considered to support multiple nano-machines communicating with a receiver. With MoTDMA, the transmission of different nano-machines is arranged to occur within different time slots, and the information conveyed from a specific nano-transmitter is then recovered from the corresponding time-slots. Furthermore, multipleaccess molecular communications can be implemented via Molecule Division Multiple Access (MDMA) [6]. With this multiple-access technique, every nano-machine is employed a particular type of molecules or a set of unique types of molecules for it to transmit information to a receiver. Hence, MDMA uses molecular types to distinguish different nanomachines.

As in the conventional radio-based communications, multiple-access communications often experiences interference, referred to in general as Multiple-Access Interference (MAI). This demands particular attention in DMC's transceiver design, because of the nature of DMC, where interference can hardly be avoided via signaling design, such as, spreading codes in MCDMA. Hence, multiple-access scheme and corresponding receiver design in DMC become highly challenging. In the context of multiple-access DMC, there are a range of detection schemes introduced and studied in references, e.g., $[1,3,5,6]$. Specifically for the MCDMA considered in [1], since the multiple-access scheme is based on On-Off Keying (OOK) modulation, the authors developed a chip-based detection scheme with the adaptive threshold obtained by referring to the concentrations of previous chips. In [3], the detection algorithm proposed in [7] was introduced for MoTDMA detection, which is also a threshold-based detector, with the threshold directly set to the number of molecules of the same type that are received during the pre- 
vious impulse period. In [5], three genetic detection methods were considered, namely the roulette-wheel selection, stochastic universal sampling and tournament selection for artificial neuronal networks. Finally, [6] considered to use different types of molecules to support multiple-access transmission. Correspondingly, the ligand-receptors matched to the different types of molecules are used to aid information detection.

In the conventional radio-based wireless communications, there is a Fast Frequency-Hopping $M$-ary Frequency Shift Keying (FFH-MFSK) scheme [8,9], which exploits multiple frequencies to simultaneously support both frequency-hopping and data transmission of multiple users. Based on the observation that frequencies in conventional radio-based communications are analogous to the different types of molecules in DMC, in this paper, we design a Molecular Type Spread MoSK (MTS-MoSK) scheme to allow DMC to simultaneously support multiple nano-machines. In MTS-MoSK DMC systems, MTS is implemented by assigning different nanomachines unique spreading codes. For a given nano-machine to send data, it first signatures the data using its unique spreading code, yielding the outputs that control the release of different types of molecules. At the receiver, different types of molecules and their numbers (densities) are measured, which are used to recover the data sent by these nano-machines with the aid of their spreading codes. In this paper, we investigate two types of low-complexity detection schemes [10], namely the Maximum Selection assisted Majority Vote Detector (MSMVD) and the Equal Gain Combining (EGC) assisted Detector (EGCD). When MS-MVD is employed, some candidate molecular types are first identified, followed by the molecular-type de-spreading and majority voting. By contrast, with EGCD, molecular-type de-spreading is first carried out. Then, the sum of concentration of each type is calculated, based on which final decision is made to give the estimate to the transmitted data. Both detection approaches have the advantage that no threshold is required, which is usually difficult to be set to a near-optimum value, because of the Brownian motion of molecules and severe ISI in DMC.

In this paper, we also analyze the error performance of the MTS-MoSK DMC systems. Specifically, when MS-MVD is employed, we derive a lower-bound for the Bit Error Rate (BER) of MTS-MoSK DMC systems. By contrast, when EGCD is employed, we derive the approximate BER of MTS-MoSK DMC systems, when both the Poisson approximation [11-13] and Gaussian approximation [14,15] are considered, respectively. Finally, the error performance of MTS-MoSK DMC systems is investigated with the aid of analytical results, Monte-Carlo simulation and particle-based simulation. It is demonstrated that the performance results obtained from analytical results and that from simulations agree with each other, which valid our analysis. The error performance of MTS-MoSK DMC systems is also compared with that of the on-off keying (OOK)-assisted DMC system, where each nano-machine is exclusively supported by one type of molecules. Furthermore, we demonstrate the impact of different parameters on the error performance of MTSMoSK DMC systems, showing the design trade-off between performance and complexity.

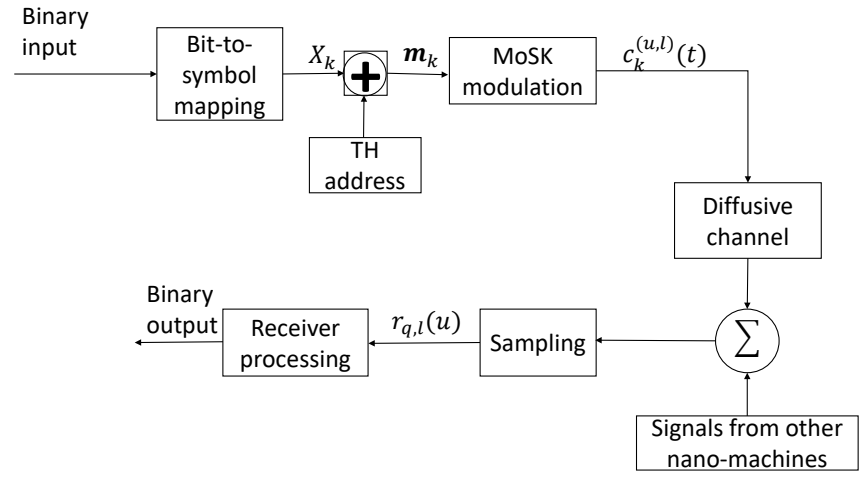

Fig. 1. System diagram showing the components of the MTS-MoSK DMC systems.

The contribution of the paper can be summarized as follows.

- A MTS-MoSK multiple-access scheme is proposed for DMC to support multiple nano-machines simultaneously communicating with a receiver.

- Two low-complexity detection schemes, namely MSMVD and EGCD, are proposed for information detection in MTS-MoSK DMC systems.

- An BER lower-bound is derived for the MTS-MoSK DMC systems with MS-MVD, and the approximate BER expressions are derived, respectively, based on the Poisson- and Gaussian approaches for the MTS-MoSK DMC systems with EGCD.

- The error performance of MTS-MoSK DMC systems is investigated based on both analytical results and simulations. Both model- and particle-based simulations are invoked for performance studies. Furthermore, the error performance of the MTS-MoSK DMC system with EGCD is compared with that of the OOK-assisted DMC system.

The remainder of this paper is arranged as follows. Section II introduces the MTS-MoSK DMC system model. In Section III, the principles of two detection schemes are introduced. Section IV details the analysis of error performance. Performance results are demonstrated in Section V. Finally, the main conclusions from research are summarized in Section VI.

\section{SYSTEM MODEL}

The MTS-MoSK DMC system proposed has the structure as shown in Fig. 1. The details about the system structure and operations are provided in the following subsections.

\section{A. Description of Transmitter and Channel Model}

We assume a MTS-MoSK DMC system, which employs $M$ types of information molecules to supports $K \leq M$ nanomachines communicating with a common access point (AP). We assume that the locations of nano-machines and AP are fixed. For simplicity, all nano-machines are assumed to have a similar distance from AP. However, we should note that in practice, this may be the case in a molecular sensor network (MSN), where multiple sensors communicate with a fusion center, deployed for monitoring an event. The transmitter of 
a nano-machine in the MTS-MoSK DMC system is shown as the above part of Fig. 1. Assume that $M$ types of molecules are employed by each of the nano-machines for information transmission. Then, $b=\log _{2} M$ bits per symbol can be delivered by a nano-machine. Assume that $T_{s}$ is the symbol-duration, which is divided into $L=T_{s} / T_{h}$ chips of each having the duration of $T_{h}=T_{s} / L$ seconds referred to as chip-duration. We assume that the transmissions of $K$ nano-machines are synchronous at chip-level. In practice, this can be achieved via synchronizing the transmissions of different nano-machines with the pilot signals periodically sent from AP. During a symbol-duration of $T_{s}$ seconds, $b$ bits of information to be transmitted by the $k$ th nano-machine is first mapped to a $M$ ary symbol expressed as $X_{k} \in\{0,1, \ldots, M-1\}$, as shown in Fig. 1. Let the MTS code of the $k$ th nano-machine be expressed as $\boldsymbol{a}_{k}=\left[a_{k}^{(0)}, a_{k}^{(1)}, \ldots, a_{k}^{(L-1)}\right], k=1,2, \ldots, K$, where $a_{k}^{(i)} \in[0, M-1]$. In practice, the MTS codes should be designed and assigned to nano-machines in favor of distinguishing the symbols transmitted by different nano-machines. As shown in Fig. 1, the $M$-ary symbol $X_{k}$ is signed by the $k$ th nano-machine's MTS code, with the operation expressed as

$$
\begin{aligned}
\boldsymbol{m}_{k}= & {\left[m_{k}^{(0)}, m_{k}^{(1)}, \ldots, m_{k}^{(L-1)}\right] } \\
= & X_{k} \cdot \mathbf{1}_{(1 \times L)} \oplus \boldsymbol{a}_{k} \\
= & {\left[X_{k} \oplus a_{k}^{(0)}, X_{k} \oplus a_{k}^{(1)}, \ldots, X_{k} \oplus a_{k}^{(L-1)}\right], } \\
& k=1,2, \ldots, K
\end{aligned}
$$

where $\oplus$ is the addition operation in the Galois field GF $(M)$ [16], and $\mathbf{1}_{(1 \times L)}$ is an all-one row vector of length $L$. After the signature operation, it can be known that the elements of $\boldsymbol{m}_{k}$ have the integer values in $[0, M-1]$, which can be directly processed by the $M$-ary MoSK modulation block as seen in Fig. 1, to emit the corresponding types of molecules within the $L$ chip-durations.

For example, assume that there are two nano-machines in the MTS-MoSK DMC system employing $M=8$ types of molecules and using $L=6$ chips per symbol for information transmission. Let the first and second nano-machines' MTS codes be $\boldsymbol{a}_{1}=[4,3,7,6,2,5]$ and $\boldsymbol{a}_{2}=[2,0,1,6,4,7]$, and their data symbols to be transmitted be $X_{1}=5$ and $X_{2}=3$, respectively. Then, to transmit $X_{1}$ and $X_{2}$, they are first extended to the $L$-length vectors of $X_{1} \cdot \mathbf{1}_{(1 \times L)}=$ $[5,5,5,5,5,5]$ and $X_{2} \cdot \mathbf{1}_{(1 \times L)}=[3,3,3,3,3,3]$, respectively. Then, by executing the addition operation with $\boldsymbol{a}_{1}$ and $\boldsymbol{a}_{2}$, respectively, in the Galois field $G F(8)$, we obtain two vectors of $\boldsymbol{m}_{1}=X_{1} \cdot \mathbf{1}_{(1 \times L)} \oplus \boldsymbol{a}_{1}=[1,6,2,3,7,0]$ and $\boldsymbol{m}_{2}=$ $X_{2} \cdot \mathbf{1}_{(1 \times L)} \oplus \boldsymbol{a}_{2}=[1,3,2,5,7,4]$, where a value represents the type of molecules to be activated within the corresponding chip-duration. Note that in $\boldsymbol{m}_{1}$ and $\boldsymbol{m}_{2}$, the colored elements illustrate that the transmissions from two nano-machines collide with each other and generate interference, as they emit the same type of molecules within the same chip-duration.

We assume that the $M$ types of molecules belong to a family of the isomers, which hence have a similar diffusion coefficient denoted by $D$ when propagating within a fluid medium [17]. Then, after the MoSK modulation, if a molecular type is activated within a given chip-duration, a pulse of molecules

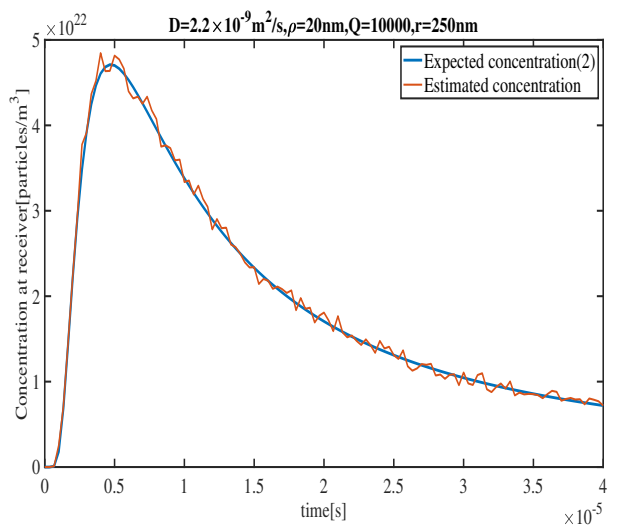

Fig. 2. Comparison between the expected concentration given by Fick's law of (2) and the concentration obtained from the average of 1000 particle-based simulations, when assuming that one pulse of molecules is emitted at $t=0$.

of this type is emitted at the beginning of the chip. Let at the $l$ th chip of the $u$ th symbol-duration, the $q$ th molecular type is activated by the $k$ th nano-machine. Then, following the Fick's diffusion law $[18,19]$, the concentration of molecules observed by AP at time $t$ for the $q$ th type of molecules emitted by the $k$ th nano-machine can be formulated as

$$
\begin{aligned}
& c_{k, q}^{(u, l)}(t)=\frac{A}{\left[4 \pi D\left(t-u T_{s}-l T_{h}\right)\right]^{3 / 2}} \\
& \quad \times \exp \left(\frac{-r^{2}}{4 D\left(t-u T_{s}-l T_{h}\right)}\right), t \geq(u L+l) T_{h}
\end{aligned}
$$

where $Q$ is the number of molecules emitted per pulse and $r$ is the distance between a transmit nano-machine and AP.

Given a molecular pulse with $Q=10000$ molecules emitted at $t=0$, Fig. 2 compares the expected concentration of (2), which is a given function, and the estimated concentration obtained from the average of 1000 particle-based simulations. It is shown that the estimated concentration agrees well with the expected concentration given by Fick's law. However, the estimated concentration still slightly fluctuates around the expected concentration, which is due to the randomness of particles' Brownian motion. Note that, according to [20], the desired signal in DMC is given by the expected value, such as, the expected concentration of (2), while the difference between the actual and expected values is unexpected and treated as noise.

From the characteristics of diffusion process, we can easily conceive that there are different kinds of interference in the MTS-MoSK DMC system. First, as shown in many references, such as in $[21,22]$, the same type of molecules transmitted corresponding to different symbols may overlap with each other, generating ISI. Second, there is background noise generated by the randomness of molecules' Brownian motion. Third, when a MTS-MoSK DMC system supports multiple nano-machines simultaneously transmitting signals to a common AP using the same $M$ types of molecules, the signals transmitted by these nano-machines in the current chip-duration interfere with each other. Furthermore, the molecules transmitted previously in the adjacent chips by the different nano-machines also interfere the signals transmitted in the current chip. These kinds of 
interference generated by the interfering nano-machines can be referred to as multiple-access interference (MAI). Therefore, in order to achieve reliable communication in MTS-MoSK DMC system, the detector operated at AP should be carefully designed.

\section{B. Observations Obtained by Receiver}

After a pulse of molecules is transmitted at the beginning of a chip, it is feasible for the receiver at AP to sample at the expected peak concentration, which can be derived from (2) and occurs at $t_{d}=r^{2} /(6 D)$. Assume that this is the case in this paper, meaning the assumption of $T_{h}>t_{d}$. The concentration sampled within the $l$ th chip of the $u$ th symbolduration can be expressed as

$$
\begin{aligned}
r_{q, l}(u)= & r_{q}\left(t=u T_{s}+l T_{h}+t_{d}\right), \\
& l=0,1, \ldots, L-1 ; u=0,1, \ldots ; \\
& q=0,1, \ldots, M-1
\end{aligned}
$$

When taking account of the Brownian motion noise, ISI from the same type of molecules transmitted previously, and the MAI from the other nano-machines, the received signal $r_{q, l}(u)$ can be expressed as

$$
\begin{aligned}
r_{q, l}(u)= & \sum_{k=1}^{K} \sum_{i=0}^{\min \{I, u L+l\}} \ell_{k, q}^{u L+l-i} c_{k, q}\left(i T_{h}+t_{d}\right) \\
& +n_{q}\left(u T_{s}+l T_{h}+t_{d}\right) \\
= & \sum_{k=1}^{K} \sum_{i=0}^{\min \{I, u L+l\}} \ell_{k, q}^{u L+l-i} c_{k, q}(i)+n_{q, l}(u)
\end{aligned}
$$

where the ISI length is assumed to span $I$ chip-durations, $\ell_{k, q}^{i}$ is a logic value, which is ' 1 ', if the $q$ th type of molecules is activated by the $k$ th nano-machine in the $i$ th chip, or is ' 0 ', otherwise. In (4), $c_{k, q}(i)$ is the expected concentration of the $q$ th type of molecules at $t=i T_{h}+t_{d}$, i.e., $c_{k, q}\left(i T_{h}+t_{d}\right)$, when an impulse of $A$ molecules of this type was sent at $t=0$. It is worth noting that here only the component with $i=0$ contributes to the detection of the desired signal, while all the other components corresponding to $i \neq 0$ generate interference. Finally in (4), $n_{q, l}(u)$ is the counting noise introduced due to the transmission of the $q$ th type of molecules, which can be approximated as the Gaussian noise [22], with the probability density function (PDF) expressed as

$$
n_{q, l}(u) \sim \mathcal{N}\left(0, \frac{1}{V} \sum_{k=1}^{K} \sum_{i=0}^{\min \{I, u L+l\}} \ell_{k, q}^{u L+l-i} c_{k, q}(i)\right)
$$

where $V$ is the volume of the space that the receiver used to measure the concentration of information molecules. From (5), we can conceive that the noise is due to the transmission of signals. In other words, whenever a signal for conveying information is transmitted, there is added noise power, and higher signal power also gives higher noise power. Hence, the noise in DMC is very different from that in the conventional radio frequency communications, where background noise is not related to transmitted signals, but added at receiver.

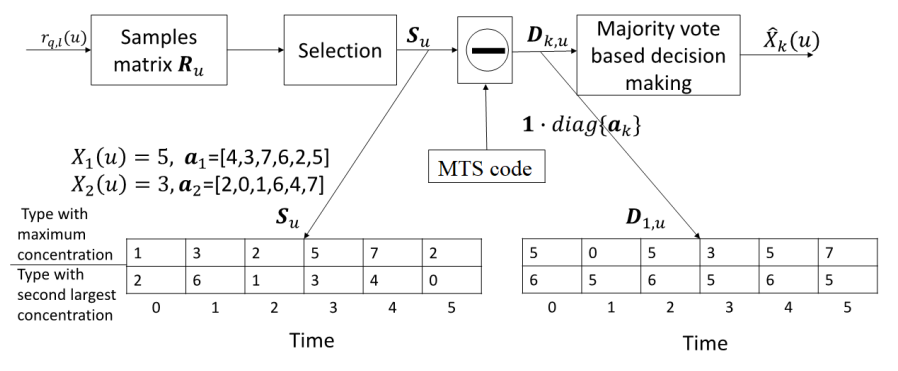

Fig. 3. Schematic block diagram for the MS-MVD.

\section{Signal Detection In MTS-MoSK DMC Systems}

From (3) we are inferred that during a symbol-duration, there are in total $M L$ observations available for detecting the symbols transmitted by the $K$ nano-transmitters during this symbol-duration. For convenience of description of our proposed detectors, these $M L$ observations obtained during the $u$ th symbol-duration are arranged as an observation matrix $\boldsymbol{R}_{u}$, which has $M$ rows corresponding to the $M$ types of molecules and hence the $M$ possible symbols, and $L$ columns representing the $L$ chips within the $u$ th symbol-duration. The $(q, l)$ th element of $\boldsymbol{R}_{u}$, i.e., $r_{q, l}(u)$, represents the concentration sample of the $q$ th type of molecules in the $l$ th chipduration of the $u$ th symbol, which is given in (4). Explicitly, the observation matrix $\boldsymbol{R}_{u}$ can be expressed as

$$
\boldsymbol{R}_{u}=\left[\begin{array}{cccccc}
r_{1,1}(u) & r_{1,2}(u) & \cdots & r_{1, l}(u) & \cdots & r_{1, L}(u) \\
r_{2,1}(u) & r_{2,2}(u) & \cdots & r_{2, l}(u) & \cdots & r_{2, L}(u) \\
\vdots & \vdots & \ddots & \vdots & \ddots & \vdots \\
r_{q, 1}(u) & r_{q, 2}(u) & \cdots & r_{q, l}(u) & \cdots & r_{q, L}(u) \\
\vdots & \vdots & \ddots & \vdots & \ddots & \vdots \\
r_{M, 1}(u) & r_{M, 2}(u) & \cdots & r_{M, l}(u) & \cdots & r_{M, L}(u)
\end{array}\right]
$$

Based on the observation matrix $\boldsymbol{R}_{u}$, below we introduce two detection approaches to detect the $u$ th symbols received from the $K$ nano-machines. The first approach implements the Maximum Selection followed by Majority Vote based Detection, which is the MS-MVD. By contrast, the second detection approach is designed in the principle of Equal-Gain Combining (EGC), which is the EGCD. Let us first describe the MS-MVD in detail.

\section{A. Maximum Selection Assisted Majority Vote Detection}

The MS-MVD can be well explained with the aid of the schematic block diagram shown in Fig. 3. This detector includes two main operations. The first main operation is the Maximum Selection (MS), which selects a number of entries that have the largest concentration from each of the $L$ columns of $\boldsymbol{R}_{u}$. The second main operation is the Majority Vote based Detection (MVD), which makes the decision of a symbol to be detected based on the number of times that a specific symbol appears, and the symbol appears the most number of times is decided to be the desired one detected.

In detail, the principle of the MS-MVD can be described as follows with reference to Fig. 3. First, from each of the $L$ columns in the observation matrix $\boldsymbol{R}_{u}, K$ elements having the 
highest concentration values are selected, the corresponding row indices of these selected elements are used to form a $(K \times L)$ candidate matrix $\boldsymbol{S}_{u}$, as shown in Fig. 3 for the case of $K=2$. In $S_{u}$, the first row accommodates the largest elements, the second row stores the second largest elements, and so on. Note that, the reason why the $K$ largest elements in each column are chosen is that the number of nano-machines supported is $K$ and hence, each column has at most $K$ active types.

The above MS scheme has the advantage that no threshold is required for selection, as threshold is sometimes hard to determine due to the lack of channel knowledge. However, it has the disadvantage that some inactive types may be selected, as $K$ elements have to be selected from each column, while there is possibility that two or more nano-machines may activate the same type of molecules during one chip, as shown in the example below (1). Hence, although our performance results for the MS-MVD in Section V are obtained by applying the above MS scheme, below we provide some discussion about the possible schemes modified from the above selection scheme. One modified selection scheme is the joint maximumthreshold selection (JMTS). With the JMTS scheme, based on $\boldsymbol{R}_{u}$, the receiver first forms a $(K \times L)$ candidate matrix $\boldsymbol{S}_{u}$ as the above described, i.e., by selecting the $K$ largest elements from each of the $L$ columns of $\boldsymbol{R}_{u}$. Then, each of the selected elements in $\boldsymbol{S}_{u}$ is compared with a threshold $T h$. If the element value is lower than $T h$, the corresponding element is emptied or counted as an erasure. Otherwise, if the element value is higher than $T h$, the element is left as it is. During the following type de-spreading operation and MVD, the empty elements will not be processed. The JMTS scheme is depended on the threshold setting, which is sometimes hard to determine, as above mentioned. One approach for the JMTS approach to circumvent this problem is to compare only a given number of the smallest elements in $S_{u}$ to the threshold. The objective of this improved approach is to retain the activated elements in the candidate matrix but remove the inactive elements from the candidate matrix with a high probability.

After $\boldsymbol{S}_{u}$ is obtained, the detector proceeds to the molecular type de-spreading operation by exploiting the knowledge about the MTS code assigned to the nano-machine being detected. Specifically for the $k$ th nano-machine, using its MTS code $\boldsymbol{a}_{k}$, the molecular type de-spreading procedure generates a $(K \times L)$ detection matrix expressed as

$$
\boldsymbol{D}_{k, u}=\boldsymbol{S}_{u} \ominus\left(\mathbf{1}_{(K \times L)} \cdot \operatorname{diag}\left\{\boldsymbol{a}_{k}\right\}\right), k=1,2, \ldots, K
$$

where $\mathbf{1}_{(K \times L)}$ is an all-one matrix with $K$ rows and $L$ columns. Therefore, $\mathbf{1}_{(K \times L)} \cdot \operatorname{diag}\left\{\boldsymbol{a}_{k}\right\}$ just copies the $k$ th nano-machine's MTS code $\boldsymbol{a}_{k}$ to each of the $K$ rows. In (6), the symbol $\ominus$ represents the element-by-element subtraction operation between $\boldsymbol{S}_{u}$ and $\mathbf{1}_{(K \times L)} \cdot \operatorname{diag}\left\{\boldsymbol{a}_{k}\right\}$ in the Galois field $\mathrm{GF}(M)$. As shown in Fig. 3, the detection matrix obtained by de-spreading $S_{u}$ using the first nano-machine's MTS code is expressed as $\boldsymbol{D}_{1, u}$.

Finally, based on the detection matrix $D_{k, u}$, the estimate to the $u$ th symbol sent by the $k$ th nano-machine is decided in the principle of majority vote, which makes the decision in favor of the symbol appearing most frequently in the

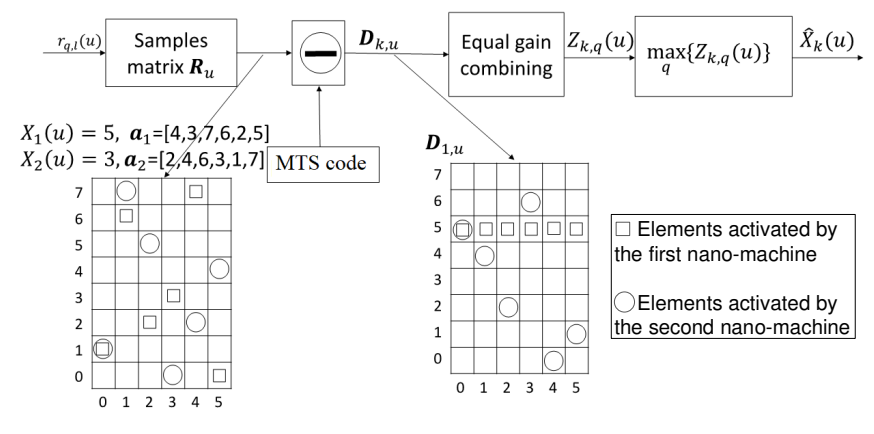

Fig. 4. Schematic block diagram showing the EGC assisted detection.

detection matrix $\boldsymbol{D}_{k, u}$. However, if there are two or more than two symbols having the same number of times appearing in $D_{k, u}$, the detector can randomly select one from them to represent the estimate to the received symbol. Specifically for the example considered, as shown in Fig. 3, symbol ' 5 ' appears the most number of times. Hence, according to the majority vote rule, $\hat{X}_{1}(u)=5$ is the estimated symbol received from the first nano-machine. Similarly, the symbol sent by the second nano-machine can be estimated, which is $\hat{X}_{2}(u)=3$, as expected.

The complexity of MS-MVD can be analyzed as follows. After the observation matrix $\boldsymbol{R}_{u}$ is prepared, for each of the $L$ columns, the detector needs to identify the $K$ largest among the $M$ types of molecules. When the quick-sort algorithm [23] is used, the average number of comparisons required to sort $M$ real numbers is $2 M \ln M$. It is known that the number of comparisons required to find the maximum among $M$ real numbers using binary-search is $(M-1)$. Hence, when $K$ is small, lower number of computations than the quicksort algorithm may be obtained by using direct comparison to find the $K$ largest among $M$ real numbers, which requires $K(M-[K+1] / 2)$ comparisons. Hence, to form the candidate matrix $S_{u}, A_{c}=2 L M \ln M$ (in average) or $A_{c}=K L(M-[K+1] / 2)$ comparisons are required, when the quick-sort algorithm or direct comparison is employed. Note that, the above operations are common for all nanomachines. By contrast, after obtaining the candidate matrix, the following computations for deriving the detection matrices and the decision making are specific to individual nanomachines. Specifically, for the $k$ th nano-machine, $K L$ number of element-by-element subtraction in $\operatorname{GF}(M)$ is required for molecular type de-spreading. Finally, finding the number of times of the $M$ possible elements appearing in $\boldsymbol{D}_{k, u}$ and then selecting the one with the highest number of times require about $K L+M$ operations. Therefore, the total operations for detecting a symbol of one nano-machine is about $A_{c} / K+2 K L+M$.

\section{B. Equal-Gain Combining (EGC) Assisted Detection}

The principle of the equal-gain combining assisted detection (EGCD) can be well understood with the aid of Fig. 4. As done in the MS-MVD, the receiver first forms the observation matrix $\boldsymbol{R}_{u}$ based on the concentrations of the $M$ types of molecules 
and of the $L$ chips. In Fig. 4, those marked elements in $\boldsymbol{R}_{u}$ represent the elements activated by the $K=2$ nano-machines. These active elements contain both signals and noise as well as interference. By contrast, those un-filled elements in $\boldsymbol{R}_{u}$ contain only noise and interference. As shown in Fig. 4, to detect the symbol received from the $k$ th nano-machine, the observation matrix $\boldsymbol{R}_{u}$ is de-spreaded using the MTS code $\boldsymbol{a}_{k}$ of the $k$ th nano-machine, yielding the detection matrix $\boldsymbol{D}_{k, u}$. To be more specific, after the type de-spreading, the $(q, l)$ th element $r_{q, l}(u)$ in $\boldsymbol{R}_{u}$ is moved to the location $\left(q \ominus a_{k}(l), l\right)$ of $D_{k, u}$, i.e.,

$$
\begin{array}{r}
d_{\left(q \ominus a_{k}(l), l\right)}^{(k)}(u)=r_{q, l}(u), q=0,1, \ldots, M-1 ; \\
l=0,1, \ldots, L-1 ; k=1,2, \ldots, K
\end{array}
$$

As an example, in Fig. 4, when $\boldsymbol{R}_{u}$ is de-spreaded using the first nano-machine's MTS code $\boldsymbol{a}_{1}=(4,3,7,6,2,5)$, yielding the detection matrix $D_{1, u}$. It can be seen that all the $L=6$ elements activated by the first nano-machine are de-spreaded to the fifth row, inferring that the received symbol is $\hat{X}_{1}(u)=$ 5 . By contrast, the elements activated by the undesired nanomachine scatter randomly over the matrix, forming MAI.

Based on the detection of $\boldsymbol{D}_{k, u}$, then, EGC can be carried out to form $M$ decision variables as

$$
\begin{gathered}
Z_{q}^{(k)}(u)=\sum_{l=0}^{L-1} d_{(q, l)}^{(k)}(u), \quad q=0,1, \ldots, M-1 ; \\
k=1,2, \ldots, K
\end{gathered}
$$

for each of $u=0,1, \ldots$ Finally, the decision on the symbol to be detected is made by choosing the largest among $\left\{Z_{0}^{(k)}(u), Z_{1}^{(k)}(u), \ldots, Z_{M-1}^{(k)}(u)\right\}$, and its subscript represents the detected symbol. This decision making procedure can be described as

$$
\hat{X}_{k}(u)=\arg \max _{q}\left\{Z_{q}^{(k)}(u)\right\}, k=1,2, \ldots, K
$$

The complexity of the EGCD can be analyzed as follows. The $M \times L$ elements of observation matrix $\boldsymbol{R}_{u}$ are directly operated in the de-spreading process. To be more specific, the detector operates the $G F(M)$ subtraction between the elements' locations and desired nano-machine's MTS code on the element-by-element basis, which transforms the observation matrix $\boldsymbol{R}_{u}$ to the detection matrix $\boldsymbol{D}_{k, u}$ by assigning every element a new location. Hence, the total number of operations in the de-spreading procedure is $(M \times L)$. Note that, because the first stage of EGCD is operated with a single MTS code, all the operations in the whole detection process are for one nano-machine. After the detection matrix $\boldsymbol{D}_{k, u}$ is attained, according to the principle of EGCD, the sum of the elements in each of the $M$ rows is required to be calculated. For each row, there are $(L-1)$ additions. Therefore, to generate the decision variables $Z_{q}^{(k)}(u), M(L-1)$ additions are required. Finally, finding the largest one from the $M$ decision variables needs $(M-1)$ operations. Consequently, when considering all the operations, the total number of computations required for detecting a symbol of one nano-machine using EGCD is approximately $2 M L$.

\section{ERror Probability ANALYSis}

In this section, we attempt to analyze the error performance of the MTS-MoSK DMC systems. In our analysis, we assume that the $M$-ary symbols transmitted by $K$ nanomachines are randomly selected with a uniform probability of $P(0)=P(1)=\cdots=P(M-1)=1 / M$, and that the random MTS codes with each of their elements obeying the discrete uniform distribution in $[0, M-1]$ are employed. Let us first analyze the MTS-MoSK DMC system with MS-MVD.

\section{A. Error Probability of MS-MVD: A Lower-Bound}

Due to the ISI and counting noise, it is extremely hard to mathematically analyze the error rate of the MTS-MoSK DMC system with MS-MVD. Therefore, we analyze for a lower bound by assuming that there is no ISI and also that the SNR is sufficiently high, so that there are no false-alarm and miss identifications of the active elements in the candidate matrix $S_{u}$. In this case, an erroneous detection occurs only because of the interference among nano-machines.

Without any loss of generality, let us assume that the 1st nano-machine is the reference one, whose transmitted symbol $X_{1}=0$ is detected. Hence, in the detection matrix $D_{1, u}$ of size $(K \times L)$, there should be $L$ and only $L$ elements being 0 . Erroneous detection occurs only when there is one or more than one symbol other than symbol 0 appearing $L$ times in $D_{1, u}$ and appearing once in each column.

Since both data symbols and MTS codes are randomly and uniformly distributed, the probability that a given symbol value other than 0 , such as $i \in\{1,2, \ldots, M-1\}$, appears in a column is

$$
P_{A}=1-\left(1-\frac{1}{M}\right)^{K-1}
$$

As the events with all the columns are independent, the probability that the same symbol $i$ appears $L$ times in $\boldsymbol{D}_{1, u}$ is

$$
P_{A}^{L}=\left[1-\left(1-\frac{1}{M}\right)^{K-1}\right]^{L}
$$

Furthermore, it can be shown that the probability that there are $j$ symbols other than symbol 0 , each of which appears $L$ times in $\boldsymbol{D}_{1, u}$ is

$$
\begin{aligned}
P(j)= & \left(\begin{array}{c}
K-1 \\
j
\end{array}\right)\left(P_{A}^{L}\right)^{j}\left(1-P_{A}^{L}\right)^{K-1-j}, \\
& j=0,1, \ldots, K-1
\end{aligned}
$$

When the above event occurs, there are $j+1$ symbols appearing the same $L$ times in $\boldsymbol{D}_{1, u}$, and the detector has to randomly choose one of them as the estimate $\hat{X}_{1}$ to the symbol $X_{1}$ transmitted by the reference nano-machine. Hence, the average symbol error probability (SEP) is

$$
P_{s}=1-\sum_{j=0}^{K-1} \frac{1}{j+1} \times P(j)
$$

Furthermore, following the principle of the $M$-ary modulation, the bit error rate (BER) is given by [24]

$$
P_{b}=\frac{M / 2}{M-1} P_{s}
$$


which is a lower-bound for the MTS-MoSK DMC system with MS-MVD.

\section{B. Error Probability of EGCD}

To analyze the error probability of the MTS-MoSK DMC systems with EGCD, below we consider two approaches to model the distributions of the elements in the detection matrices of $K$ nano-machines, namely, Poisson-approach and Gaussian-approach. In our analysis, again, we assume that the first nano-machine is the reference one, whose transmitted symbol $X_{1}(u)=0$ is to be detected. Hence, our analysis is based on $D_{1, u}$. For simplicity, the index 1 of the reference nano-machine is ignored in the analysis.

First, let us consider the Poisson approach. Assume that the volume of the space used by the receiver to measure the molecular concentration is $V$. Then, when the ISI symbols and the symbols sent by the $K$ nano-machines during the $u$ th symbol-duration are given, the number of molecules of $D_{m, l}(u)=V d_{m, l}(u)$ obtained based on (7) obeys the Poisson distribution with the PMF expressed as

$$
\begin{gathered}
f_{D_{m, l}(u)}\left(n \mid \mathcal{X}_{I}(u), X_{1}(u)=0\right)=\frac{\lambda_{m, l}^{n}(u) e^{-\lambda_{m, l}(u)}}{n !}, \\
n=0,1, \ldots,
\end{gathered}
$$

where $\mathcal{X}_{I}(u)$ contains all the ISI symbols from the $K$ nanomachines as well as the $K-1$ interfering symbols sent by the $(K-1)$ interfering nano-machines during the $u$ th symbolduration, $\lambda_{m, l}(u)$ is the mean value of $D_{m, l}(u)$ under the above conditions. From (7), we can conceive that

$$
\lambda_{m, l}(u)=\lambda_{q, l}(u), l=0,1, \ldots, L-1
$$

with $q=m \oplus a_{1}^{(l)}$, where $\lambda_{q, l}(u)$ can be obtained from (4) as

$$
\begin{aligned}
\lambda_{q, l}(u) & =E\left[V r_{q, l}(u)\right] \\
& =V \sum_{k=1}^{K} \sum_{i=0}^{\min \{I, u L+l\}} \ell_{k, q}^{u L+l-i} c_{k, q}(i)
\end{aligned}
$$

When the EGCD is employed, the $M$ decision variables for the reference nano-machine are given by (8). Since the sum of Poisson distributed random variables is still Poisson distributed, the PMF of $D_{m}(u)$ can be expressed as

$$
\begin{gathered}
f_{D_{m}(u)}\left(n \mid \mathcal{X}_{I}, X_{1}(u)=0\right)=\frac{\Lambda_{m}^{n}(u) e^{-\Lambda_{m}(u)}}{n !} \\
n=0,1, \ldots ; m=0,1, \ldots, M-1
\end{gathered}
$$

where $\Lambda_{m}(u)=\sum_{l=0}^{L-1} \lambda_{m, l}(u)$.

With the PMFs of the decision variables prepared, the conditional probability that the $u$ th symbol of the reference nano-machine is correctly detected can be derived as

$$
\begin{aligned}
P_{c}^{(1)} & \left(\mathcal{X}_{I}, X_{1}(u)=0\right) \\
= & P\left(D_{0}(u)>D_{1}(u), \ldots, D_{0}(u)>D_{M-1}(u)\right) \\
= & \sum_{n_{0}=0}^{\infty} P\left(D_{0}(u)=n_{0}\right) \prod_{m=1}^{M-1} P\left(D_{m}(u)<n_{0}\right) \\
= & \sum_{n_{0}=0}^{\infty} f_{D_{0}(u)}\left(n_{0} \mid \mathcal{X}_{I}, X_{1}(u)=0\right) \\
& \times \prod_{j=1}^{M-1} P\left(D_{j}(u)<n_{0} \mid \mathcal{X}_{I}, X_{1}(u)=0\right) \\
= & \sum_{n_{0}=0}^{\infty}\left[\frac{\Lambda_{0}^{n_{0}}(u) e^{-\Lambda_{0}(u)}}{n_{0} !}\right] \cdot \prod_{j=1}^{M-1}\left[\sum_{n_{m}=0}^{n_{0}-1} \frac{\Lambda_{m}^{n_{m}}(u) e^{-\Lambda_{m}(u)}}{n_{m} !}\right]
\end{aligned}
$$

As $D_{m}(u)$ are discrete random variables, it is possible that $D_{0}(u)$ is just one of the several maximums. In this cases, the detector randomly and uniformly selects one of the maximums for the final detection. Correspondingly, the conditional probability that the $u$ th symbol of the reference nano-machine is correctly detected is

$$
\begin{gathered}
P_{c}^{(2)}\left(\mathcal{X}_{I}, X_{1}(u)=0\right)=\sum_{j=1}^{M-1} \frac{1}{j+1}\left(\begin{array}{c}
M-1 \\
j
\end{array}\right) \\
\times P\left(D_{0}(u)=D_{i_{1}}(u), \ldots, D_{0}(u)=D_{i_{j}}(u) ;\right. \\
\left.D_{0}(u)>D_{i_{j+1}}(u), \ldots, D_{0}(u)>D_{i_{M-1}}(u)\right)
\end{gathered}
$$

where $i_{j} \in\{1,2, \ldots, M-1\}$. Following (19), we can derive $P_{c}^{(2)}\left(\mathcal{X}_{I}, X_{1}(u)=0\right)$ as

$$
\begin{aligned}
P_{c}^{(2)} & \left(\mathcal{X}_{I}, X_{1}(u)=0\right)=\sum_{j=1}^{M-1} \frac{1}{j+1}\left(\begin{array}{c}
M-1 \\
j
\end{array}\right) \\
& \times \sum_{n_{0}=0}^{\infty} P\left(D_{0}(u)=n_{0}\right) \prod_{m_{1}=i_{1}}^{i_{j}} P\left(D_{m_{1}}(u)=n_{0}\right) \\
& \times \prod_{m_{2}=i_{j+1}}^{i_{M-1}} P\left(D_{m_{2}}(u)<n_{0}\right) \\
= & \sum_{j=1}^{M-1} \frac{1}{j+1}\left(\begin{array}{c}
M-1 \\
j
\end{array}\right) \sum_{n_{0}=0}^{\infty}\left[\frac{\Lambda_{0}^{n_{0}}(u) e^{-\Lambda_{0}(u)}}{n_{0} !}\right] \\
& \times \prod_{m_{1}=i_{1}}^{i_{j}}\left[\frac{\Lambda_{m_{1}}^{n_{0}}(u) e^{-\Lambda_{m_{1}}(u)}}{n_{0} !}\right] \\
& \times \prod_{m_{M}=i_{j+1}}^{i_{M-1}}\left[\sum_{n_{m}=0}^{n_{0}-1} \frac{\Lambda_{m_{2}}^{n_{m}}(u) e^{-\Lambda_{m_{2}}(u)}}{n_{m} !}\right]
\end{aligned}
$$

When we combine (19) and (21), a compact form expression 
can be obtained, which is

$$
\begin{aligned}
P_{c} & \left(\mathcal{X}_{I}, X_{1}(u)=0\right) \\
= & \sum_{j=0}^{M-1} \frac{1}{j+1}\left(\begin{array}{c}
M-1 \\
j
\end{array}\right) \sum_{n_{0}=0}^{\infty}\left[\frac{\Lambda_{0}^{n_{0}}(u) e^{-\Lambda_{0}(u)}}{n_{0} !}\right] \\
& \times \prod_{m_{1}=i_{1}}^{i_{j}}\left[\frac{\Lambda_{m_{1}}^{n_{0}}(u) e^{-\Lambda_{m_{1}}(u)}}{n_{0} !}\right] \\
& \times \prod_{m_{2}=i_{j+1}}^{i_{M-1}}\left[\sum_{n_{m}=0}^{n_{0}-1} \frac{\Lambda_{m_{2}}^{n_{m}}(u) e^{-\Lambda_{m_{2}}(u)}}{n_{m} !}\right]
\end{aligned}
$$

where we set $i_{0}=0$ and in this case, $i_{0+1}=1$. Note that, in the case of $D_{0}(u)=0$, the only event for possible correct detection is $D_{0}(u)=D_{1}(u)=\ldots=D_{M-1}(u)=0$, which yields a correct symbol probability of $1 / M$.

The average correct symbol probability can be obtained by considering all the possible symbols in $\mathcal{X}_{I}$, which can be expressed as

$$
P_{c}=\frac{1}{M^{\left|\mathcal{X}_{I}\right|}} \sum_{\mathcal{X}_{I}} P_{c}\left(\mathcal{X}_{I}, X_{1}(u)=0\right)
$$

However, this is usually impossible to compute, as the ISI is in general very long in DMC. To simplify (23), we only consider the average with the symbols sent by the $K-1$ nano-machines during the $u$ th (current) symbol-duration, while the ISI symbols sent before the $u$ th symbol period are averaged out using their statistics. Specifically, when the Poisson-approximation is employed, we approximate $D_{m, l}(u)$ as a Poisson distributed random variable with the PMF given by

$$
\begin{gathered}
f_{D_{m, l}(u)}\left(n \mid X_{1}(u)=0, X_{2}(u), \ldots, X_{K}(u)\right) \\
=\frac{\lambda_{m, l}^{n}(u) e^{-\lambda_{m, l}(u)}}{n !}, n=0,1, \ldots,
\end{gathered}
$$

where $\lambda_{m, l}(u)$ still follows (16), but with $\lambda_{q, l}(u)$ given by

$$
\begin{array}{r}
\lambda_{q, l}(u) \\
=V \sum_{k=1}^{K}\left[\sum_{i=0}^{\min \{I, l\}} \ell_{k, q}^{u L+l-i} c_{k, q}(i)+\frac{1}{M} \sum_{i=L}^{\min \{I, u L+l\}} c(i)\right] \\
=V\left[\sum_{k=1}^{K} \sum_{i=0}^{\min \{I, l\}} \ell_{k, q}^{u L+l-i} c_{k, q}(i)+\frac{K}{M} \sum_{i=L}^{\min \{I, u L+l\}} c(i)\right]
\end{array}
$$

where the second term in the bracket is a constant obtained by assuming that the data symbols obey the uniform distribution in $[0, M-1]$.

With the above approximation, (23) can now be re-written as

$$
\begin{aligned}
P_{c}= & \frac{1}{M^{K-1}} \sum_{X_{2}(u), \ldots, X_{K}(u) \in\{0, \ldots, M-1\}} P_{c}\left(X_{1}(u)=0,\right. \\
& \left.X_{2}(u), \ldots, X_{K}(u)\right)
\end{aligned}
$$

Correspondingly, the BER of the MTS-MoSK DMC system with EGCD can be obtained from the formula [24]

$$
P_{b}=\frac{M / 2}{M-1}\left(1-P_{c}\right) \text {. }
$$

In the context of the Gaussian approach, $D_{m, l}(u)$ is approximated as a Gaussian distributed random variable with the PDF given by

$$
D_{m, l}(u) \sim \mathcal{N}\left(\lambda_{m, l}(u), \lambda_{m, l}(u)\right)
$$

where $\lambda_{m, l}(u)$ is the same as the one in (15). Furthermore, since the sum of Gaussian distributed random variables is still Gaussian distributed, the PDF of $D_{m}(u)=\sum_{l=0}^{L-1} D_{m, l}(u)$ is

$$
D_{m}(u) \sim \mathcal{N}\left(\Lambda_{m}(u), \Lambda_{m}(u)\right), m=0,1, \ldots, M-1
$$

where $\Lambda_{m}(u)$ is the same as the one in (18). Then, following the derivation of (19) and remembering that $D_{m}(u)$ are continue random variables, we can obtain the conditional correct symbol probability as

$$
\begin{aligned}
P_{c} & \left(\mathcal{X}_{I}, X_{1}(u)=0\right)=\int_{-\infty}^{\infty} f_{D_{0}(u)}(x) d x \\
& \times \prod_{m=1}^{M-1} \int_{-\infty}^{x} f_{D_{m}(u)}(y) d y
\end{aligned}
$$

where $f_{D_{m}(u)}(x)$ is the Gaussian PDF given by (29). Upon applying the PDFs of (29) into the above equation, it can be shown that

$$
\begin{aligned}
P_{c}\left(\mathcal{X}_{I}, X_{1}(u)=0\right)= & \int_{-\infty}^{\infty} \frac{1}{\sqrt{2 \pi} \sigma_{0}} \exp \left(-\frac{\left[x-\mu_{0}\right]^{2}}{2 \sigma_{0}^{2}}\right) d x \\
& \times \prod_{m=1}^{M-1}\left(1-Q\left[\frac{x-\mu_{m}}{\sigma_{m}}\right]\right)
\end{aligned}
$$

where $Q(t)$ is the Gaussian Q-function defined as $Q(t)=$ $(2 \pi)^{-1 / 2} \int_{t}^{\infty} e^{-t^{2} / 2} d t$, and by definition,

$$
\begin{gathered}
\mu_{m}=\sigma_{m}^{2}=\Lambda_{m}(u)=\sum_{l=0}^{L-1} \lambda_{m, l}(u), \\
m=0,1, \ldots, M-1
\end{gathered}
$$

Note that, all $\mu_{m}$ and $\sigma_{m}^{2}$ are dependent on $\mathcal{X}_{I}$ and $X_{1}(u)=0$.

Continuing from (30), the average correct symbol probability can in theory be obtained from (23). Furthermore, if we apply the $\lambda_{q, l}(u)$ as shown in (25), we can obtain for the average correct symbol probability a relatively simplified expression as shown in (26). Finally, the BER is given by (27). However, even in this case, the computation for the BER is extreme. As shown in (31), there are $M$-ple integrals to compute for every set of the given $(K-1)$ symbols of $\left\{X_{2}, \ldots, X_{K}\right\}$, not need to mention that $\left\{X_{2}, \ldots, X_{K}\right\}$ have in total $M^{K-1}$ combinations. For this sake, in Section $\mathrm{V}$, we only evaluate the error probability of EGCD, when nanomachines' MTS codes are fixed, i.e., without considering the randomness of MTS codes.

\section{Performance Results and Discussion}

In this section, the error performance of the MTS-MoSK DMC systems with respectively the MS-MV and EGCD is demonstrated against the number of molecules emitted per bit expressed as $A_{b}$, when various cases are considered. As in MTS-MoSK DMC systems, each symbol carries $b=\log _{2} M$ bits, the total number of molecules emitted for transmitting one 


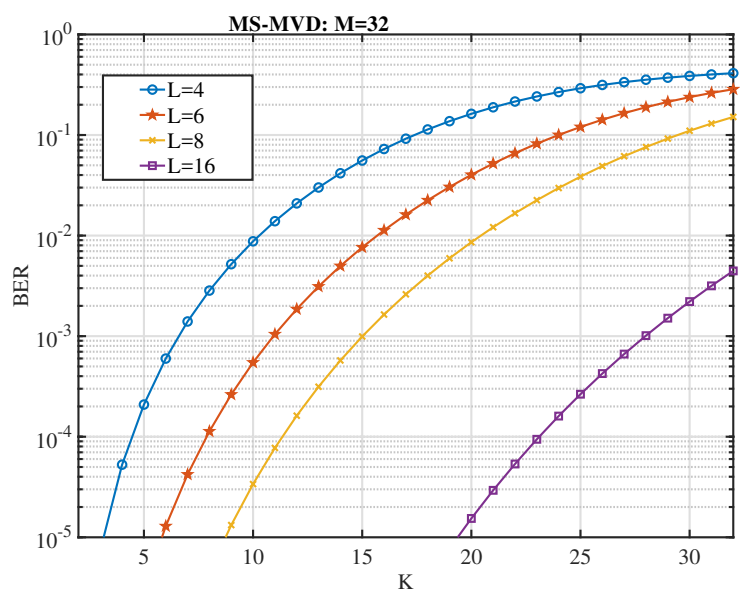

Fig. 5. Lower-bound BER versus $K$ of the number of nano-machines for the MTS-MoSK DMC system with MS-MVD, when given $M=32$ and different number of chips per symbol.

symbol is $A_{s}=b \times A_{b}$. However, due to the introduction of MTS, each symbol is transmitted using $L$ chips by activating $L$ molecular pulses. Hence, the number of molecules emitted per pulse is $A_{h}=A_{s} / L$. Additionally, we note that $A_{b}$ in the figures is shown in $\mathrm{dB}$ values given by $10 \log _{10} A_{b}$.

In our performance studies, unless specifically noted, there are some parameters set to fixed values, which are $D=2.2 \times$ $10^{-9} \mathrm{~m}^{2} / \mathrm{s}$ and $V=4 \pi \rho^{3} / 3$ with $\rho=20 \mathrm{~nm}$. The bit-duration is set to $T_{b}=6 \times 10^{-5} \mathrm{~s}$, hence the symbol-duration is $T_{s}=$ $b \times\left(6 \times 10^{-5}\right) s$, and furthermore, the chip-duration is $T_{h}=$ $T_{s} / L$. Additionally, considering the effect of ISI, we evaluate the effective length of a transmitted molecular pulse according to

$$
I \triangleq \arg _{i}\left\{\frac{c_{h(i)}}{c_{h(o)}} \leq 0.1 \%\right\}
$$

Assume that a pulse of molecules is emitted at $t=0, c_{h(o)}$ in the above expression is the peak concentration obtained within the first chip-duration, while $c_{h}(i)$ is the concentration sampled at the $i$ th chip-duration. Expression (33) means that only the concentration with the strength above $0.1 \%$ of the peak concentration is considered. Additionally, all the MTS codes employed in our studies are random codes.

Fig. 5 and Fig. 6 demonstrate the potential BER performance of the MTS-MoSK DMC systems with MS-MVD, which were evaluated from the lower-bound formulas derived in Section IV-A. Specifically in Fig. 5, we investigate the idealized error performance (lower-bound BER) versus $K$ of the number of nano-machines supported by the MTSMoSK DMC system, when $M=32$ and $L=4,6,8,16$, respectively. Explicitly, the error performance of the MTSMoSK DMC system degrades with the increase of the number of nano-machines supported. As shown in Fig. 5, as the number of chips used to transmit a symbol increases, the error performance continuously improves. The reason behind the observation is that when more chips per symbol is used, it becomes harder for the interferers to form the same number of entries as the reference nano-machine does. In other words, when the number of chips per symbol increases, the inter-

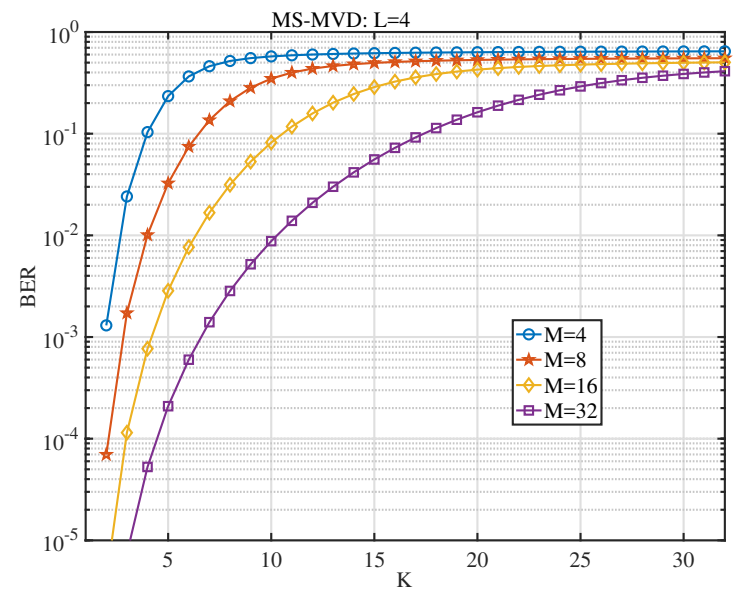

Fig. 6. Lower-bound BER versus $K$ of the number of nano-machines for the MTS-MoSK DMC system with MS-MVD, when given $L=4$ and different modulation level.

ference between any two nano-machines, i.e., MAI, reduces, hence resulting in the improvement of error performance. Note that for a given chip-duration, data rate reduces as the number of chips per symbol increases, which is also an explanation for the improvement of error performance.

Fig. 6 illustrates the impact of the number of molecular types on the BER performance of the MTS-MoSK DMC system with MS-MVD, when $L=4$ is assumed. From the principles of MTS-MoSK DMC, we can know that when $M$ increases, the modulation level increases and the number of bits conveyed per symbol increases. From the results of Fig. 6, we can observe that the BER performance improves, as $M$ is increased from $M=4$ to $M=32$, respectively, corresponding to 2 to 5 bits per symbol. This observation conforms to the noble characteristics of MoSK modulation for interference mitigation. When given a value of $L$ and a given value of $K$, as the value of $M$ increases, there are more entries in the observation matrix $\boldsymbol{R}_{u}$ for the interfering elements to distribute. Hence, the MAI decreases, as the value of $M$ increases, therefore resulting in the improvement of BER performance.

In Figs. 7 and 8, we compare the BER performance of the MTS-MoSK DMC systems with EGCD, which are obtained respectively from analytical approaches and simulation approaches. Note that, in term of the channel models, the simplified $\lambda_{q, l}(u)$ as shown in (25) is used to generate the number of molecules for both analysis and simulation. Furthermore, to make the performance evaluation and simulation manageable, the MTS codes are first randomly generated and then fixed for obtaining both analytical and simulation results.

In detail, in Fig. 7, we demonstrate the BER performance of the MTS-MoSK DMC systems employing EGCD, when various symbol-duration $T$ and hence different data rates are considered. To obtain the analytical results in Fig. 7, the Poisson approach introduced in Section IV-B is employed, and the results obtained by this approach are compared with that obtained from simulation. Note that, the MTS codes for the $K=$ 4 nano-machines are $\boldsymbol{a}_{1}=[3,10,14,13,9,11,5,12], \boldsymbol{a}_{2}=$ $[15,3,11,10,6,7,5,0], \boldsymbol{a}_{3}=[8,6,9,5,12,4,10,13], \boldsymbol{a}_{4}=$ 


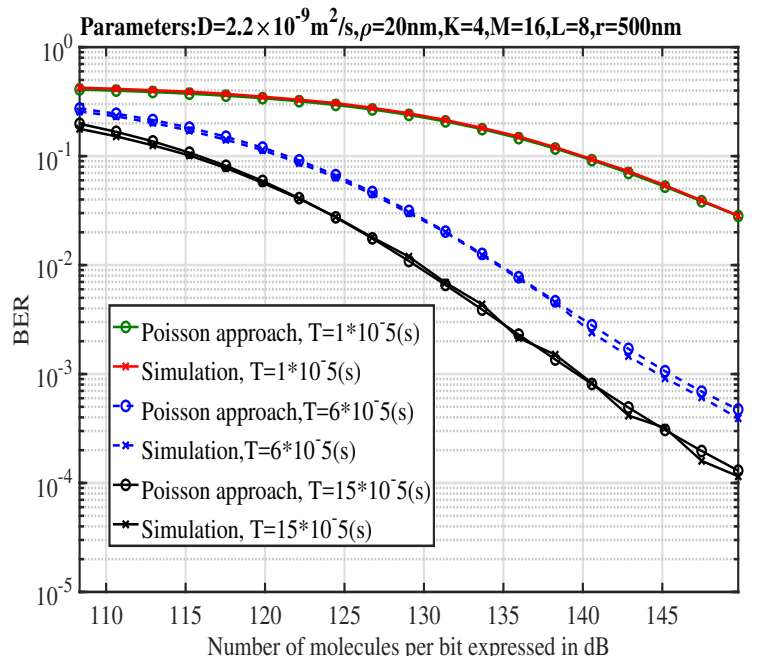

Fig. 7. BER performance of MTS-MoSK DMC systems with EGCD, where the analytical results were evaluated based on the Poisson-approach.

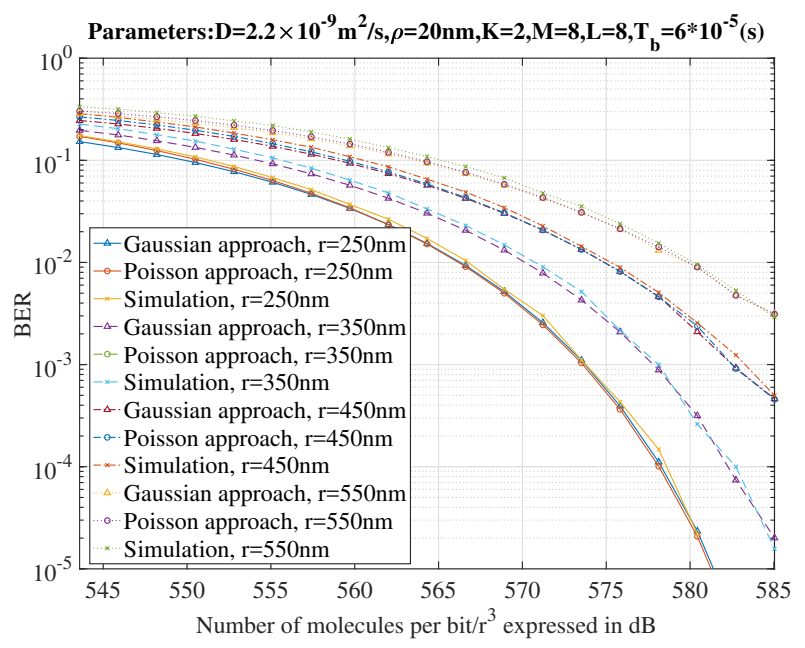

Fig. 8. BER performance of MTS-MoSK DMC systems with EGCD where the analytical results were evaluated based on the Poisson-approach and Gaussian approach, respectively.

$[4,15,3,5,12,6,1,0]$, and the other parameter values are detailed with the figure. As the results in Fig. 7 show, first, the analytical results agree well with the simulation results. Thus, the Poisson analytical approach is valid for performance study of MTS-MoSK DMC systems. Second, when the symbolduration increases or data rate decreases, the BER performance of the MTS-MoSK DMC systems with EGCD improves, as the result of ISI reduction due to the increase of symbol-duration.

Fig. 8 compares the BER performance obtained from the analytical results based respectively on the Poisson approach and Gaussian approach, and that obtained by simulations. We assume that $K=2$ nano-machines are invoked, and their MTS codes are $\boldsymbol{a}_{1}=[2,0,5,6,7,3,4,1]$ and $\boldsymbol{a}_{2}=$ $[0,4,2,1,5,7,6,3]$. The results in Fig. 8 show that the analytical results obtained from both the approaches are very similar, and their agree well with the simulation results, although the analytical results slightly under estimate the simulation

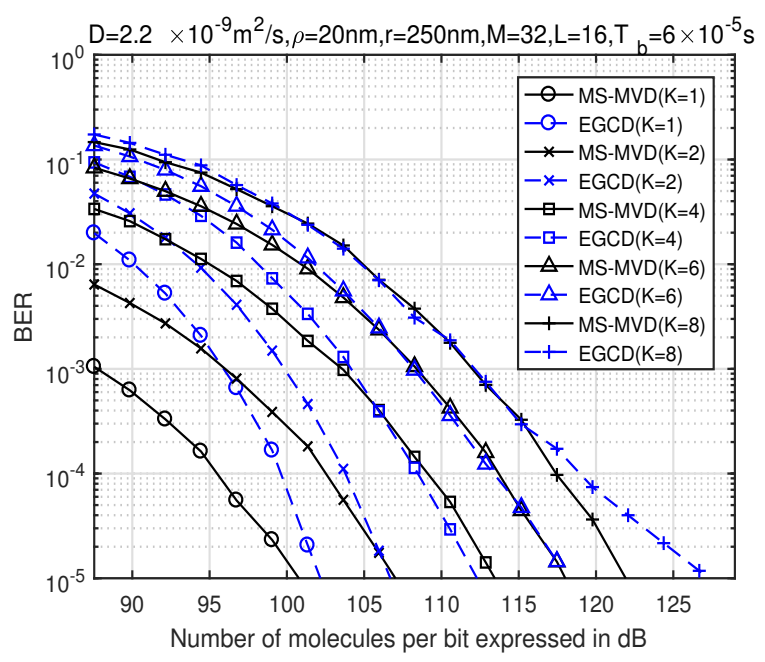

Fig. 9. Comparison of BER performance of the MTS-MoSK DMC systems employing MS-MVD and EGCD, respectively.

results. Therefore, both the Poisson approach and Gaussian approach can be employed for analyzing the performance of MTS-MoSK DMC systems, and our analytical results obtained in Section IV are valid. Additionally, from Fig. 8 we can clearly observe that the BER performance of the MTS-MoSK DMC systems degrades as the transmission distance increases. This is because as the transmission distance increases, the ISI increases, and hence the BER performance degrades.

In Fig. 9, we compare the BER performance of the MTSMoSK DMC systems employing respectively the MS-MVD and EGCD, when different numbers of nano-machines as shown in the figure are supported, while all the other parameters are fixed with the values shown on the top of the figure. From Fig. 9 we can have the following observations. First, for both the MS-MVD and EGCD, the BER performance declines, as the number of nano-machines supported increases, as the result of increased MAI. Second, MS-MVD outperforms EGCD, when $A_{b}$ is relatively small. This becomes more declared, as the number of nano-machines supported becomes smaller. In other words, the MS-MVD is more desirable, when the number of nano-machines is lower, in particular, if $A_{b}$ is small. By contrast, when $A_{b}$ is relatively big, such as higher than $105 \mathrm{~dB}$ for $K=4$, the BER performance achieved by EGCD becomes better than that attainable by MS-MVD. Therefore, MS-MVD is beneficial for operation in the case that the number of molecules emitted per pulse is relatively small, but is less effective than EGCD in MAI dominant environments. To be more specific, when $A_{b}$ is small, the system performance is dominated by the counting noise, which depends on the transmitted signals. When EGCD is employed, the decision valuables are the sums of the row elements of matrix $D_{k, u}$, as seen in Fig. 4. These operations enhance the influence of noise. By contrast, by selecting the largest ones from each column, the MS-MVD is capable of efficiently mitigating the effect of noise, making it outperform the EGCD in low $A_{b}$ region. On the other side, if $A_{b}$ is sufficiently high, the system performance becomes dominated by MAI. As the sum operation in EGCD is able to smooth the MAI but the 


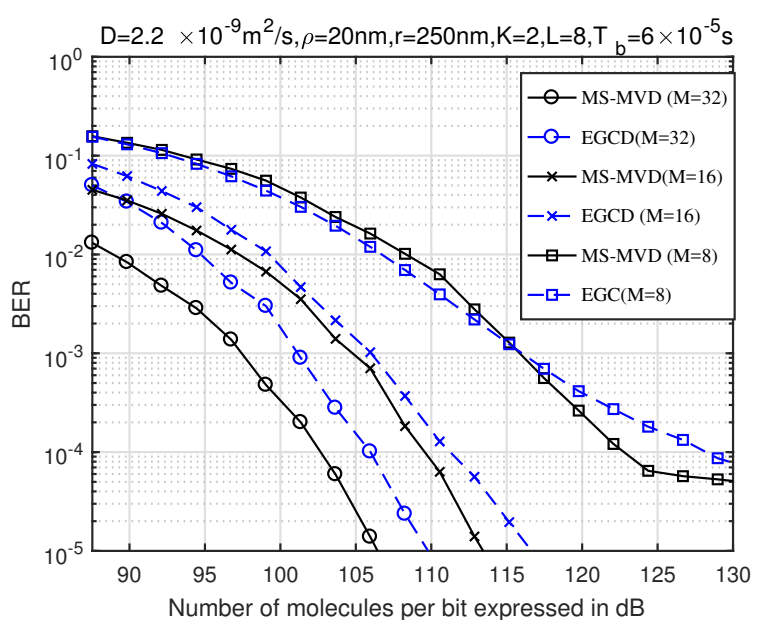

Fig. 10. Comparison of BER performance of the MTS-MoSK DMC systems employing respectively MS-MVD and EGCD, when different numbers of molecular types are invoked.

MS-MVD is unable to, the EGCD performs better than the MS-MVD. This observation can be further evidenced by the results with the increase of the number of nano-machines. As shown in Fig. 9, for a given and low $A_{b}$ value, the gap between the BER achieved by EGCD and that by MS-MVD becomes smaller, as $K$ increases.

Fig. 10 compares the BER performance of MS-MVD with that of the EGCD with respect to different modulation levels, namely, $M=8,16$ and 32 . We can see that when the modulation level $M$, i.e., the number of molecular types, increases, the BER performance achieved by both detection schemes improves significantly. The reason behind this observation is that when $M$ increases, both the ISI and MAI reduce. Another observation we can attain from Fig. 10 is that MS-MVD becomes more promising than EGCD, as $M$ increases. As the curves in Fig. 10 show, when $M=8$, both BER curves are close to each other and when $A_{b}$ is relatively small, MS-MVD is in fact outperformed by the EGCD. By contrast, when $M$ is increased to 16 and further to 32 , the performance improvement of the MS-MVD with respect to the EGCD enhances, and the performance gap between them becomes bigger.

Given the bit-duration $T_{b}=6 \times 10^{-5} s$ and hence, the symbol-duration, Fig. 11 compares the MS-MVD with EGCD, when different values of $L$ are considered. From the results of Fig. 11, we observe that the BER performance does not monotonically improves, as the number of chips $L$ per symbol increases. As the results show, for both the MS-MVD and EGCD, $L=16$ attains the best BER performance over the main SNR region. The only exception is that the EGCD with $L=32$ achieves the best BER performance, after the $A_{b}$ is higher than about $110 \mathrm{~dB}$. The reason for the above observation is that the increase of $L$ has two-fold of effect. First, increasing $L$ is capable of averaging out (smoothing) the effect of noise and ISI. However, when $L$ increases, more molecular pulses per symbol are transmitted and for a fixed symbol-duration, the chip-duration is reduced, which therefore, result in the increase of ISI and MAI. The above

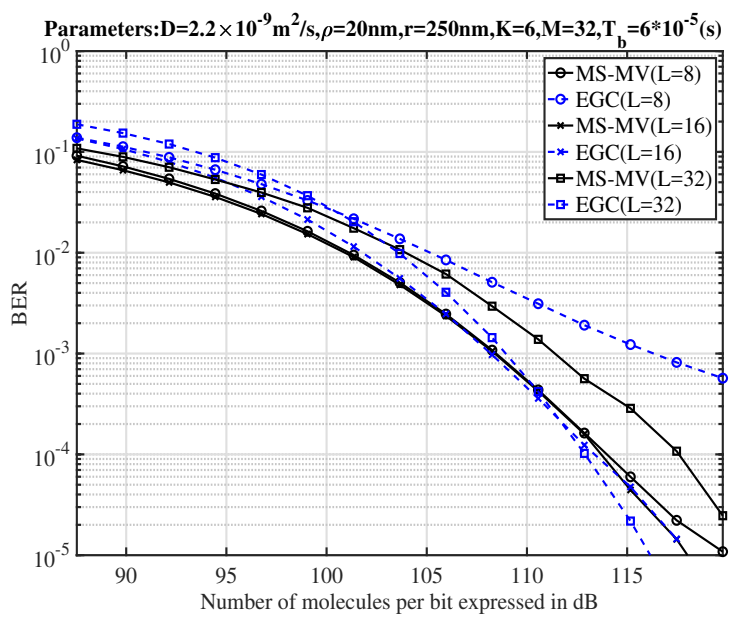

Fig. 11. Comparison of the BER performance of the MTS-MoSK DMC systems employing respectively MS-MVD and EGCD, when various number of chips per symbol is considered.

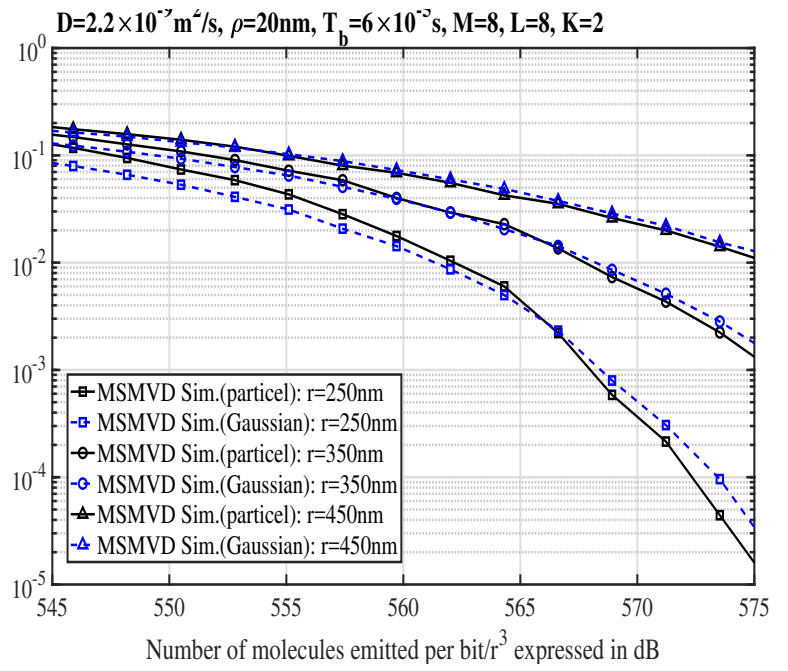

Fig. 12. BER versus the number of molecules emitted per bit normalized by $/ r^{3}$ for the MTS-MoSK DMC systems with MS-MVD, where results were obtained from the Gaussian-modeling Monte-Carlo simulations or the particle-based simulations.

observations and analysis imply that for given other parameters and a detection scheme (either MS-MVD or EGCD), there is an optimum value of $L$, which is capable of attaining the lowest BER.

Finally, in order to validate the model-based simulation and our analytical results, particle-based simulation results are compared with that obtained from the model-based simulations or/and analytical results in the following two figures. First, in Fig. 12, we compare the BER performance of the MTSMoSK DMC systems with MS-MVD obtained respectively from the Gaussian-model based Monte-Carlo simulations and the particle-based simulations. In our simulations, we assume $K=2$ nano-machines with their MTS codes of $\boldsymbol{a}_{1}=$ $[2,0,5,6,7,3,4,1]$ and $\boldsymbol{a}_{2}=[0,4,2,1,5,7,6,3]$, respectively. Furthermore, since different $r$ values are considered, as shown in Fig. 12, we use $A_{b}$ normalized by $r^{3}$, i.e., $A_{b} / r^{3}$, for the $\mathrm{X}$-axis. This is because at the sampling point of $t_{d}=$ 
$\mathrm{D}=2.2 \times 10^{-9} \mathrm{~m}^{2} / \mathrm{s}, \rho=20 \mathrm{~nm}, \mathrm{r}=250 \mathrm{~nm}, \mathrm{M}=8, \mathrm{~L}=8, \mathrm{~K}=2$

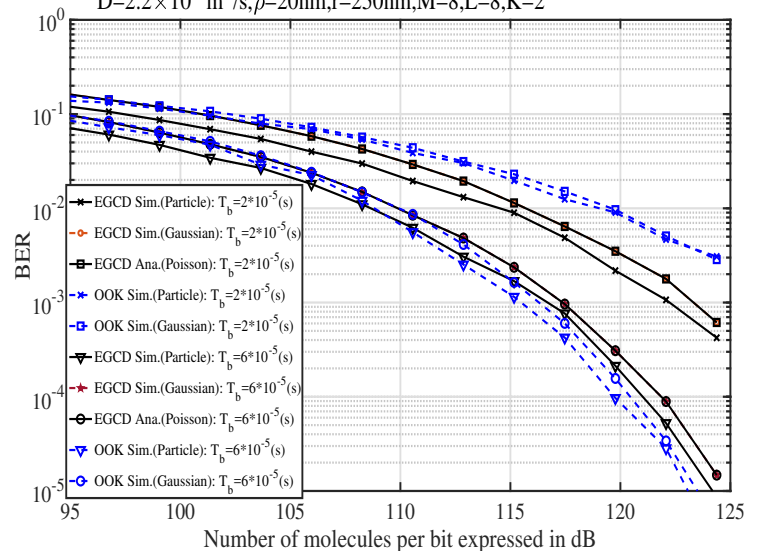

Fig. 13. Comparison of the BER versus SNR performance of the MTS-MoSK DMC systems with EGCD and that of OOK-DMC systems, where analytical results were evaluated based on the Poisson-approach and simulation results were obtained from Monte-Carlo and particle-based methods, respectively.

$r^{2} / 6 D$, the molecular density is $c_{b(o)}=\left(A_{b} / r^{3}\right)^{-1}(2 \pi e / 3)^{\frac{3}{2}}$ Hence, for a given value of $A_{b} / r^{3}$, the molecular density is independent of $r$. In this case, furthermore, the number of received molecules by a detector with volume $V$ is $V c_{b(o)}=V\left(A_{b} / r^{3}\right)^{-1}(2 \pi e / 3)^{\frac{3}{2}}$, which is also a function of $A_{b} / r^{3}$. In other words, regardless of the distance $r$ between the transmitter and receiver, the received power is the same when $A_{b} / r^{3}$ is given. Therefore, the comparison for different $r$ values is done under the same receive power. From the results we can observe that, first, the BER performance degrades when increasing $r$, as the result of increased interference. Second, the BER obtained from the model based simulations agrees well with that obtained from the particle-based simulations. Furthermore, the BER results obtained from the model-based simulations become closer to that obtained from the particlebased simulations, as the distance $r$ between transmitter and receiver increases. The reason behind is obvious. The spherical receiver (with a radius of $\rho=20 \mathrm{~nm}$ ) is modeled as a point receiver in the model based simulations, but not in the particle-based simulations. Explicitly shown in Fig. 12, the approximation to a point receiver becomes more accurate as $r$ increases, or more precisely, as $r / \rho$ increases.

In Fig. 13, we compare the BER performance of the MTS-MoSK DMC systems with EGCD obtained from different approaches, including the Poisson based analytical approach, Gaussian-based Monte-Carlo simulations and the particle-based simulations. We also assume $K=2$ nanomachines with the MTS codes of $\boldsymbol{a}_{1}=[2,0,5,6,7,3,4,1]$ and $\boldsymbol{a}_{2}=[0,4,2,1,5,7,6,3]$. Note that in the figure, the results corresponding to EGCD sim (Gaussian) fully overlap with the results corresponding to EGCD Ana (Poisson). We also compare the BER performance of the MTS-MoSK DMC systems with that of the OOK DMC systems, where each of nanomachines is exclusively supported by one type of molecules. For fair comparison, both systems are set to have the same data rate and the same power budget. The results in Fig. 13 demonstrate that when the bit-duration is $T_{b}=2 \times 10^{-5}$, implying a relatively high bit rate, the BER performance of the MTS-MoSK DMC systems with EGCD is better than that of the OOK DMC systems. By contrast, when the bit rate is relatively low corresponding to $T_{b}=6 \times 10^{-5}$, the $\mathrm{OOK}$ DMC slightly outperforms the MTS-MoSK DMC in terms of the BER performance. The reason behind is that OOK DMC does not experience MAI but suffers from ISI, which becomes severer as bit-duration becomes smaller or as data rate becomes higher. By contrast, while MTS-MoSK DMC suffers from MAI, its ISI can be much lower than OOK DMC, which becomes more noticeable as data rate increases. Hence, when data rate is relatively high, MTS-MoSK DMC is capable of achieving better BER performance than OOK DMC. On the other side, when data rate is low, resulting in that the ISI in OOK DMC is insignificant, the BER performance of OOK DMC may become better than that of MTS-MoSK DMC, as in this case the MTS-MoSK DMC may still suffer from MAI. From these observations we may deduce that MTSMoSK DMC is beneficial to supporting the multiple-access transmission requiring relatively high data rate.

Additionally, the results of Fig. 13 show that in both MTSMoSK DMC and OOK DMC, the result obtained from the particle-based simulation is slightly lower than that obtained from the Poisson-based analysis or Monte-Carlo simulation. The reason is that in the Poisson-based analysis or MonteCarlo simulation, the molecular density within the observation space is assumed to be uniform. However, in the particle-based simulation, the molecules within the observation space is nonuniform distributed, which results in a slight difference from the uniform distribution based analysis/simulation. Again, we can deduce that the order of difference is depended on the distance between transmitter and receiver relative to the size of the observation space, i.e., on the ratio of $\rho / r$. Provided that this ratio is sufficiently big, such as $\geq 10$, the model based Monte-Carlo simulations, which approximate receiver as a point, can be confidently employed for the performance estimation/prediction of DMC systems, as evidenced by both Fig. 12 and Fig. 13.

\section{CONCLUSIONS}

In order for DMC to support multiple access communications, a MTS-MoSK DMC scheme has been proposed. Correspondingly, two low-complexity detection approaches, namely MS-MVD and EGCD, have been proposed. It is shown that both detection approaches are not dependent on thresholds for detection, which are beneficial for implementation, as threshold in DMC is usually difficult to set, due to the transmission-relied noise and strong ISI. In this paper, the error performance of MTS-MoSK DMC systems has been investigated based on both analytical results and simulations. It can be shown that the analytical results are valid. Against the conventional radio-based communications where EGCD always outperforms MS-MVD, our studies demonstrate that in a DMC system supporting a small number of nano-machines relative to $L$, MS-MVD usually outperforms EGCD at relatively low SNR. When the SNR is high, MS-MVD may be outperformed by EGCD. By contrast, when a DMC system supports a big number of nano-machines relative to $L$, the BER performance achieved by both MS-MVD and EGCD is close 
to each other, although MS-MVD may slightly outperform (or be outperformed by) EGCD, depending on the SNR region observed. In term of the impact of modulation order, it is shown that the error performance improves, as the modulation order $M$ increases. Furthermore, it is shown that for a given number of molecules released per symbol and a given number of nano-machines supported, there is an optimum value for $L$, which results in the best error performance. Our future research will consider the design of promising MTS codes for MTS-MoSK DMC systems, as well as the low-complexity detection schemes with MAI cancellation capability, in order to enhance the DMC reliability and/or support more nanomachines.

\section{ACKNOWLEDGEMENTS}

The authors would like to acknowledge with thanks the anonymous reviewers for their valuable suggestions, in particular, to add the particle-based simulations.

\section{REFERENCES}

[1] Y. Zamiri-Jafarian, S. Gazor, and H. Zamiri-Jafarian, "Molecular code division multiple access in nano communication systems," in 2016 IEEE Wireless Communications and Networking Conference, April 2016, pp. $1-6$.

[2] L. Wang and A. W. Eckford, "Nonnegative code division multiple access techniques in molecular communication," in 2017 15th Canadian Workshop on Information Theory (CWIT), June 2017, pp. 1-5.

[3] S. Korte, M. Damrath, M. Damrath, and P. A. Hoeher, "Multiple channel access techniques for diffusion-based molecular communications," in SCC 2017; 11th International ITG Conference on Systems, Communications and Coding, Feb 2017, pp. 1-6.

[4] H. Tezcan, S. Oktug, and F. N. Kok, "Neural delay lines for TDMA based molecular communication in neural networks," in 2012 IEEE International Conference on Communications (ICC), June 2012, pp. 6209-6213.

[5] R. Yu, M. S. Leeson, and M. D. Higgins, "Multiple-access scheme optimisation for artificial neuronal networks," in 2014 9th International Symposium on Communication Systems, Networks Digital Sign (CSNDSP), July 2014, pp. 428-433.

[6] G. Aminian, M. Farahnak-Ghazani, M. Mirmohseni, M. Nasiri-Kenari, and F. Fekri, "On the capacity of point-to-point and multiple-access molecular communications with ligand-receptors," IEEE Transaction on Molecular, Biological and Multi-Scale Communications, vol. 1, no. 4 pp. 331-346, Dec 2015.

[7] M. Damrath and P. A. Hoeher, "Low-complexity adaptive threshold detection for molecular communication," IEEE Transactions on NanoBioscience, vol. 15, no. 3, pp. 200-208, April 2016

[8] A. Pouttu, H. Saarnisaari, and S. Glisic, "Noncoherent m MCSK-m MFSK modulation in rayleigh fading channel," in MILCOM 2006 - 2006 IEEE Military Communications conference, Oct 2006, pp. 1-5.

[9] S. Ahmed, R. G. Maunder, L. Yang, and L. Hanzo, "Iterative detection of unity-rate precoded FFH-MFSK and irregular variable-length coding," IEEE Transactions on Vehicular Technology, vol. 58, no. 7, pp. 37653770, Sep. 2009.

[10] L.-L. Yang, Multicarrier communications. John Wiley \& Sons, 2009.

[11] A. Noel, K. C. Cheung, and R. Schober, "Optimal receiver design for diffusive molecular communication with flow and additive noise," IEEE Transactions on NanoBioscience, vol. 13, no. 3, pp. 350-362, Sep. 2014.

12] A. Ahmadzadeh, A. Noel, and R. Schober, "Analysis and design of multi-hop diffusion-based molecular communication networks," IEEE Transactions on Molecular, Biological and Multi-Scale Communications, vol. 1, no. 2, pp. 144-157, June 2015.

[13] H. Arjmandi, A. Gohari, M. N. Kenari, and F. Bateni, "Diffusionbased nanonetworking: A new modulation technique and performance analysis," IEEE Communications Letters, vol. 17, no. 4, pp. 645-648, April 2013.

[14] M. U. Mahfuz, D. Makrakis, and H. T. Mouftah, "A comprehensive analysis of strength-based optimum signal detection in concentrationencoded molecular communication with spike transmission," IEEE Transactions on NanoBioscience, vol. 14, no. 1, pp. 67-83, Jan 2015.
[15] — "A comprehensive study of sampling-based optimum signal detection in concentration-encoded molecular communication," IEEE Transactions on NanoBioscience, vol. 13, no. 3, pp. 208-222, Sep. 2014

[16] D. J. Goodman, P. S. Henry, and V. K. Prabhu, "Frequency-hopped multilevel FSK for mobile radio," The Bell System Technical Journal, vol. 59, no. 7, pp. 1257-1275, Sept 1980.

[17] N. Kim and C. Chae, "Novel modulation techniques using isomers as messenger molecules for nano communication networks via diffusion,' IEEE Journal on Selected Areas in Communications, vol. 31, no. 12, pp. 847-856, 2013.

[18] L. Shi and L. Yang, "Diffusion-based molecular communications: Inter-symbol interference cancellation and system performance," in 2016 IEEE/CIC International Conference on Communications in China (ICCC), July 2016, pp. 1-6.

[19] I. Llatser, A. Cabellos-Aparicio, M. Pierobon, and E. Alarcon, "Detection techniques for diffusion-based molecular communication," IEEE Journal on Selected Areas in Communications, vol. 31, no. 12, pp. 726734, December 2013.

[20] M. Pierobon and I. F. Akyildiz, "Diffusion-based noise analysis for molecular communication in nanonetworks," IEEE Transactions on Signal Processing, vol. 59, no. 6, pp. 2532-2547, 2011.

[21] D. Kilinc and O. B. Akan, "Receiver design for molecular communication," IEEE Journal on Selected Areas in Communications, vol. 31, no. 12, pp. 705-714, December 2013.

[22] L. Meng, P. Yeh, K. Chen, and I. F. Akyildiz, "On receiver design for diffusion-based molecular communication," IEEE Transactions on Signal Processing, vol. 62, no. 22, pp. 6032-6044, Nov 2014.

[23] W. Press and et.al, Numerical Recipes in C: The Art of Scientific Computing, 2nd ed. Cambridge University Press, 1992.

[24] J. G. Proakis, Digital Communications, 5th ed. McGraw Hill, 2007.

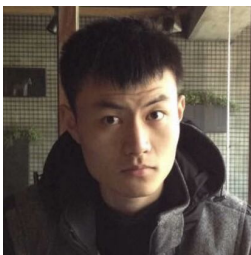

Weidong Gao received his B.Eng degree in electronic and information from the Soochow University, Suzhou, China, in 2016 and his M.Sc degree in electronic engineering from the University of Southampton, Southampton, U.K., in 2017, where he is currently pursuing the Ph.D degree. His research interest is in molecular communications, in particular, in diffusive molecular communications.

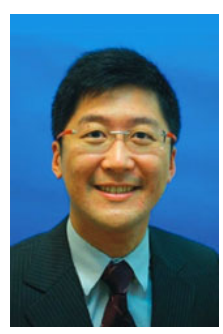

Terrence Mak is currently an Associate Professor with the Department of Electronics and Computer Science, University of Southampton, Southampton, U.K. He was graduated at Imperial College London for his PhD and a Visiting Scientist with Massachusetts Institute of Technology, Cambridge, MA, USA, in 2010. He has granted two U.S. and one U.K. patents of his engineering designs, i.e., US 16/685 090 and US 13/638 330 . He has published more than 150 papers in both conferences and journals and jointly published four books. His research areas include computer architecture design, optimization and adaptation for VLSI systems, network-on-chip, 3-D integrated circuits (ICs), and, lately, wireless-on-chip. Prof. Mak received the IET Premium Yearly Best Paper Award for Computer \& Digital Techniques in 2013, and his newly published journal based on 3DIC received the prestigious 2015 IET Computers \& Digital Techniques Premium Award. Throughout a spectrum of publications, he has awarded six best paper awards, and one nominated, from prestigious conferences, at EMBS 2005, DATE 2011, VLSI-SoC 2014, PDP 2015, EUC 2016, DATE 2018 (nominated), and ISPLED 2019. His publication at the IEEE Transactions was selected as “Top 25 Downloaded Manuscript” in 2015. 